\title{
Is Oncogene Addiction Angiogenesis-dependent?
}

\author{
J. FolkMAN AND S. RYEOM \\ The Vascular Biology Program, Department of Surgery, Children's Hospital \\ and Harvard Medical School, Boston, Massachusetts 02115
}

\begin{abstract}
Does an activated oncogene that initiates tumor growth need to remain activated to maintain the cancer phenotype? This question has been answered affirmatively by experiments in which doxycycline-regulated oncogene activation induces growth of large tumors that regress completely upon oncogene inactivation - a phenomenon called oncogene addiction. We assemble here the evidence that oncogene addiction is angiogenesis-dependent. Although activated oncogenes increase tumor cell proliferation and decrease their apoptosis, these activities are not sufficient to expand tumor mass beyond a microscopic size. Oncogenes must also induce tumor angiogenesis for expansion of tumor mass. We propose experiments to validate the "endothelial centric" hypothesis of oncogene addiction.
\end{abstract}

Rapid tumor growth in mice can be induced by implantation of tumor cells driven by an oncogene (i.e., myc or ras) under the control of a doxycycline-regulated promoter. Upon inactivation of the oncogene, tumors undergo rapid regression (Chin et al. 1999; Felsher and Bishop 1999; Brandvold et al. 2000; Jain et al. 2002; Karlsson et al. 2003; Shachaf et al. 2004). This phenomenon has been called "oncogene addiction," to indicate the need for "continued activity of a specific oncogene to maintain the cancer phenotype" (Weinstein 2002).

Growth of a tumor and maintenance of tumor mass have been thought to depend on increased tumor cell proliferation and decreased tumor cell apoptosis, both of which are oncogene-driven. However, other experimental evidence reveals that cancer cells arising from oncogene transformation of normal cells are not inherently harmful to the host (Achilles et al. 2001; Folkman and Kalluri 2004). In fact, cancer cells can remain as a microscopic-sized dormant tumor (in situ carcinoma in humans) until the dormant tumor switches to the angiogenic phenotype and is permitted to expand its tumor mass. In virtually all animal and human cancers, it is the expanding tumor mass that can potentially invade, metastasize, and eventually kill the host-not the microscopic dormant tumor. In humans, only the expanding tumor mass is potentially symptomatic and detectable by conventional imaging methods - not the microscopic dormant tumor.

In animals, expansion of tumor mass induced by an activated oncogene, and regression of tumor mass following inactivation of an oncogene, both appear as seamless processes. Once oncogene activation has occurred, tumor growth is assumed to be inevitable, and after an oncogene has been inactivated, tumor regression is believed to be virtually automatic.

We assemble here evidence that angiogenesis is a critical event in oncogene addiction. There is considerable evidence to suggest that, unless an activated oncogene can trigger the switch to the angiogenic phenotype, growth of tumor mass cannot occur. We further propose that tumor regression following oncogene inactivation may be pre- vented by deletion of an endogenous angiogenesis inhibitor engendered by oncogene inactivation. We propose, in other words, that increased tumor cell proliferation and decreased tumor cell apoptosis mediated by oncogene activation are necessary, but not sufficient to expand tumor mass. These events must be followed by sustained angiogenesis. Furthermore, tumor regression after inactivation of a specific oncogene may also require potent inhibition of angiogenesis. Although these ideas are based on an experimental model of a single angiogenesis inhibitor, it is likely that more than one endogenous angiogenesis inhibitor is operating during oncogene activation and inactivation in humans. For the purpose of organizing the data presented here, we use the term "endothelial centric" hypothesis of oncogene addiction.

\section{EXPERIMENTAL ONCOGENE ADDICTION}

Since the discovery of oncogenes and tumor suppressor genes, a fundamental question has emerged. Does the activation of an oncogene necessary for the initial development of a tumor continue to be required to maintain the cancer phenotype of that tumor (Weinstein 2002)? Chin et al. (1999) addressed this question by an elegant experiment in which the continued presence of the activated oncogene H-Ras ${ }^{\mathrm{V} 12 \mathrm{G}}$ was necessary not only to cause growth of a melanoma, but also to maintain the tumor. Doxycycline-inducible H-Ras ${ }^{\mathrm{V} 12 \mathrm{G}}$ melanomas were grown in mice lacking the tumor suppressor INK4a. Withdrawal of doxycycline down-regulated H-Ras ${ }^{\mathrm{V} 12 \mathrm{G}}$ and resulted in rapid tumor regression. Histological analysis during the initial stages of regression revealed marked apoptosis in host-derived vascular endothelial cells and in tumor cells. Furthermore, Felsher and Bishop (1999) showed that transgenic mice which conditionally expressed the myc proto-oncogene in hematopoietic cells developed T-cell lymphomas and acute myeloid leukemias. When the Myc transgene was inactivated, tumors regressed (Fig. 1). There was precipitate arrest of tumor cell proliferation followed by differentiation and apoptosis of tumor cells. In an osteogenic sarcoma model, 
A

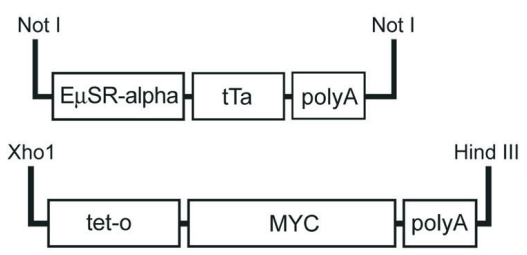

B
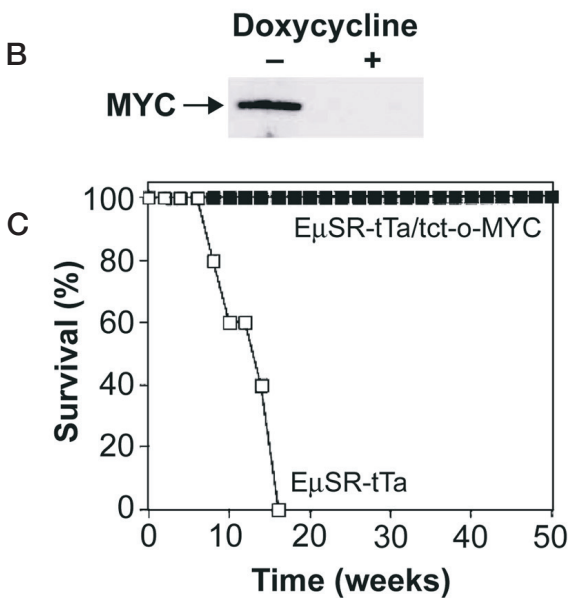

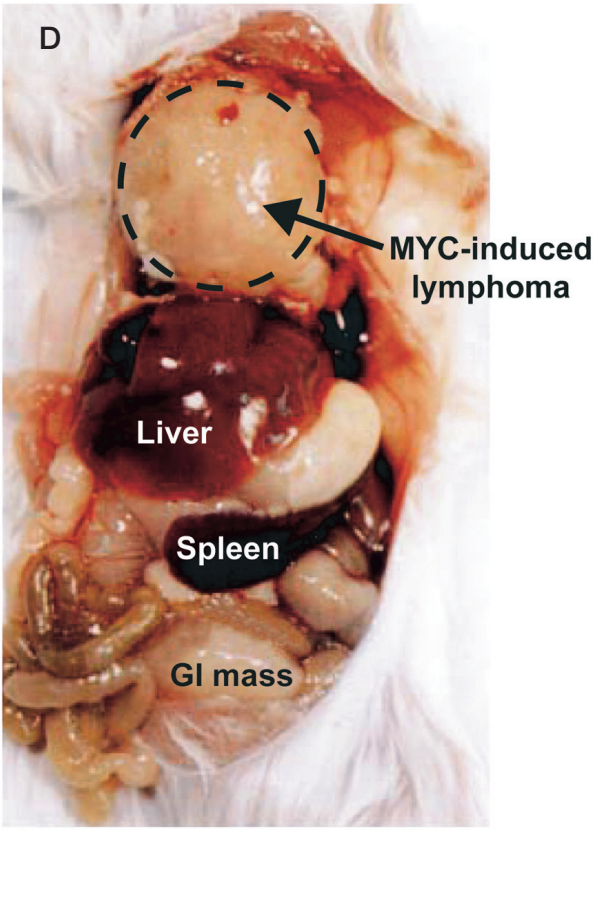

Figure 1. Conditional transgenic model for myc-induced tumorigenesis. $(A)$ Tetracycline regulatory system constructs. $(B)$ Mice transgenic for both EuSR-tTA nd tet-o-MYC conditionally express MYC protein in splenic lymphocytes. Western analysis was performed on spleen lymphocytes from transgenic mice in the absence $(-)$ or the presence $(+)$ of doxycycline in drinking water. Similar results were seen in four independent experiments. (C) Survival curves for transgenic mice. ( $\square$ ) Mice transgenic for EuSR-tTA and tet-o-MYC; (ם) mice transgenic for EuSR-tTA and tet-o-MYC with doxycycline treatment or either EuSR-tTA or tet-o-MYC alone. $n=20$ animals per group. $(D)$ Deceased animals had an enlarged thymus, spleen, liver, and mesenteric lymph nodes. (Reprinted, with permission, from Felsher and Bishop 1999.)

even brief loss of Myc for only 10 days resulted in sustained tumor regression with differentiation of osteocytes that formed histologically normal bone. Subsequent reactivation of Myc did not restore the cells' malignant properties, but induced apoptosis. Other examples have been reported that demonstrate the need for sustained activation of an oncogene for maintenance of the cancer phenotype (Weinstein 2002; Giuriato et al. 2003). In these reports, the cancer phenotype is represented by measurable or visible tumor mass, up to $1 \mathrm{~cm}^{3}$ or more.

The rapid regrowth of tumor in most reports of reactivation of an oncogene raises a second fundamental question. The rapid regrowth of tumor starting from a few "differentiated" residual tumor cells after tumor regression seems too brief a time for gene mutations to drive these cells through the classic events of tumorigenesis, which include initiation, promotion, transformation, and progression. However, epigenetic, non-mutational events could effect rapid changes in gene expression. Those genes that control the ability of tumor cells to switch to the angiogenic phenotype (Hanahan and Folkman 1996) appear capable of changing their expression rapidly enough to account for the rapid regression of tumors following inactivation of a specific oncogene, and the rapid regrowth of tumor mass after reactivation of the oncogene. The switching of angiogenesis "on" and "off" is a good candidate for a process that could swiftly mediate the growth and regression of large tumor masses.

\section{EXPANSION OF TUMOR MASS IS ANGIOGENESIS-DEPENDENT}

Since 1971, when it was first proposed that tumor growth is angiogenesis-dependent, numerous experiments have provided biological and pharmacological evidence for this concept, as well as formal genetic proof (for review, see Folkman 2000; Folkman et al. 2006; see also Kim et al. 1993; O'Reilly et al. 1994, 1997). A summary of these studies reveals that most solid tumors can form microscopic tumors up to approximately $1 \mathrm{~mm}^{3}$ without the need to recruit new blood vessels. However, further expansion of tumor mass beyond that size requires angiogenesis. Microscopic tumors incapable of switching to this angiogenic phenotype, i.e., non-angiogenic tumors, remain dormant indefinitely.

Tumor dormancy due to blocked angiogenesis is defined as a nonexpanding microscopic tumor, in which proliferation of tumor cells is balanced by apoptosis (Holmgren et al. 1995). (There are other types of tumor dormancy induced by hormone withdrawal, or by immunity, or by tumor cells in $\mathrm{G}_{0}$.)

The most compelling evidence for the angiogenic switch in human tumors is revealed by separating non-angiogenic tumor cells and angiogenic tumor cells from human tumors obtained from the operating room or from human tumor cell lines (obtained from the American Tissue Culture Collection) (Achilles et al. 2001; Udagawa et al. 
2002; Almog et al. 2006; Naumov et al. 2006). Non-angiogenic tumor cells have been found in virtually every tumor so far examined by us, including human breast cancer, colon cancer, osteosarcoma, and liposarcoma. They can make up to $\sim 20 \%$ of the tumor cell population in human tumors. Non-angiogenic tumor cells have similar proliferation rates and similar karyotypes as angiogenic tumor cells. They differ markedly in their expression of positive and negative angiogenesis regulatory proteins. For example, non-angiogenic tumor cells often express the angiogenesis inhibitor thrombospondin-1 at high levels and VEGF at moderate levels. In contrast, angiogenic tumor cells express low levels of thrombospondin-1 and increased levels of VEGF. These experiments are relevant to oncogene dependence, because they demonstrate a mechanism by which microscopic dormant tumors can suddenly and rapidly switch to an expanding tumor mass. For example, non-angiogenic tumor cells from human liposarcoma, when implanted into SCID immunodeficient mice and observed by luciferase bioluminescence, remain microscopic and dormant at $<1 \mathrm{~mm}$ diameter for a median of 133 days $\pm 2-3$ weeks, at which time virtually $100 \%$ of these non-angiogenic dormant tumors become angiogenic and grow exponentially to large tumor masses of $>1-2$ $\mathrm{cm}^{3}$ (Fig. 2) (Almog et al. 2006; Naumov et al. 2006). Before the angiogenic switch, the microscopic dormant tumors are harmless to the host, analogous to the tumors with an inactivated oncogene. After the angiogenic switch, the tumors are lethal to the mice, analogous to tumors with an activated oncogene. In other types of human tumors, the time to the angiogenic switch is different, but just as predictable. The percentage of non-angiogenic tumors that switch to the angiogenic phenotype is also predictable but differs in other tumors. For example, in a human breast cancer cell line (MDA-MB-436), approximately 80\% switch at a median of 60 days. Those that don't become angiogenic remain harmless through the remainder of the host's life. In another breast cancer (MDA-MB-415), the angiogenic switch occurs after 1 year. Transfection of a non-angiogenic tumor (i.e., osteosarcoma) with Ras will induce the angiogenic switch in virtually all tumors within 2-3 weeks (Udagawa et al. 2002).

\section{DISSOCIATION OF THE TUMOR CELL PROLIFERATIVE INDUCING FUNCTION FROM THE ANGIOGENESIS INDUCING FUNCTION OF ONCOGENES}

A common argument against angiogenesis dependence of tumor growth is that angiogenesis could simply be a secondary event that follows tumor cell proliferation. The argument goes that any oncogene activation, or in fact, any therapy that arrested tumor cell proliferation, would also arrest angiogenesis.

However, repeated studies show that tumor cell proliferation and angiogenesis can operate independently from each other. As discussed above, non-angiogenic and angiogenic human tumors have similar proliferation rates. In another study, tumor cells were treated with ionizing irradiation to render them incapable of cell division and were then implanted into the rabbit cornea; these cells still induced angiogenesis similar to nonirradiated tumor cells (Auerbach et al. 1975). A third demonstration of the dissociation of tumor cell proliferation and angiogenesis came from experiments with the SV40 large-T oncogene. When wild-type endothelial cells were transformed by the SV40-T oncogene, the resulting neoplastic cells displayed significantly increased proliferation and decreased apoptosis, as well as the other hallmarks of transformation in vitro (Arbiser et al. 1997). Furthermore, they formed microscopic, non-angiogenic, dormant tumors in vivo. These tumors were visible under low-power magnification and remained harmless throughout the life of the host. However, when the cells were transfected with a second oncogene, Ras, they switched to the angiogenic phenotype, VEGF expression and secretion increased, metalloproteinases- 2 and -9 increased, tissue inhibitor of metalloproteinase- 2 (TIMP-2) decreased, and fatal angiosarcomas developed.

Watnick et al. demonstrated that relatively low levels of H-ras in combination with the SV40 early region and hTERT result in the transformed phenotype of primary human breast and kidney epithelial cells (Volpert and Alani 2003; Watnick et al. 2003). These cells formed small dormant tumors in nude mice and were limited in further growth because of their inability to induce a vas-
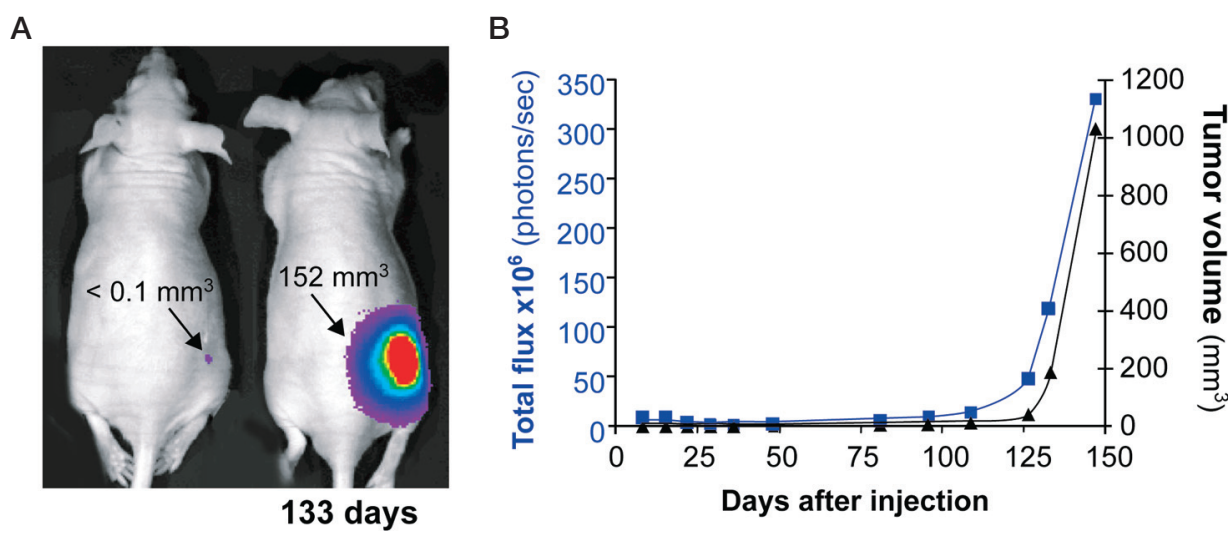

Figure 2. (A) Luciferase-labeled human liposarcoma cells that are non-angiogenic and were inoculated subcutaneously in SCID mice. Mouse on the left bears a non-angiogenic dormant tumor that is undetectable by gross examination. In the mouse on the right, the tumor has become angiogenic and is growing rapidly. $(B)$ Squares represent bioluminescence. Triangles represent palpable tumor. The tumor became palpable $\left(\sim 50-100 \mathrm{~mm}^{3}\right)$ approximately 23 days after the significant increase in bioluminescence. (Modified from Almog et al. 2006.) 
cular supply. This state of tumor dormancy could be overcome by expressing higher levels of ras which suppressed production of the angiogenesis inhibitor, thrombospondin-1. Higher levels of Ras did not significantly increase expression of VEGF.

\section{ACTIVATION OF MULTIPLE ONCOGENES POTENTIATES TUMOR ANGIOGENESIS}

Additional supporting evidence for the "endothelial centric" hypothesis of oncogene addiction was reported for human prostate cancer. After a tumor has already become angiogenic and capable of steady growth in mice, its angiogenic activity can be increased further by introducing another oncogene. The human prostate cancers PC-3 and DU-145 were transfected with the oncogene bcl-2. VEGF expression was significantly increased by 2.3-fold, and microvessel density was also significantly increased by 2-fold (Fig. 3) (Fernandez et al. 2001). Tumors derived from PC-3-bcl-2-transfected cells grew at approximately twice the rate of tumors derived from control tumor cells. Growth of human prostate cancer (PC-3) was completely suppressed in mice treated with mitomycin C, which blocked tumor cell proliferation. In contrast, prostate cancers transfected with $b c l-2$ were protected against tumor cell apoptosis induced by mitomycin $\mathrm{C}$ and therefore were completely resistant to this chemotherapeutic agent (Fig. 4). However, when bcl-2transfected prostate cancer that was resistant to mitomycin $\mathrm{C}$ was treated by an angiogenesis inhibitor, TNP470 , tumor growth was completely suppressed. TNP-470 is a synthetic analog of fumagillin (Satchi-Fainaro et al. 2004), inhibits endothelial cell proliferation at 3 logs lower than the concentration necessary to inhibit prolifer-

A
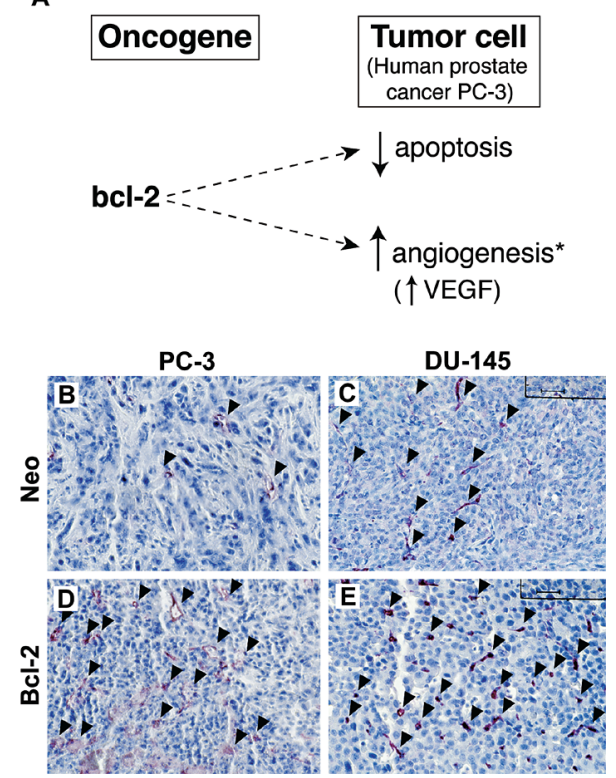

Figure 3. Representative sections showing microvessel density in human prostate cancer in SCID mice. (A) PC3-neo cells. $(B)$ PC3-bcl-2 cells. (C) DU-145 neo cells. (D) DU-145-bcl-2 cells. Tumors arising from these stably transfected cells were fixed and stained with anti-CD31 antibody to detect blood vessels. Magnification, 113x. Bars, $60 \mu \mathrm{m}$. (Reprinted, with permission, from Fernandez et al. 2001.)

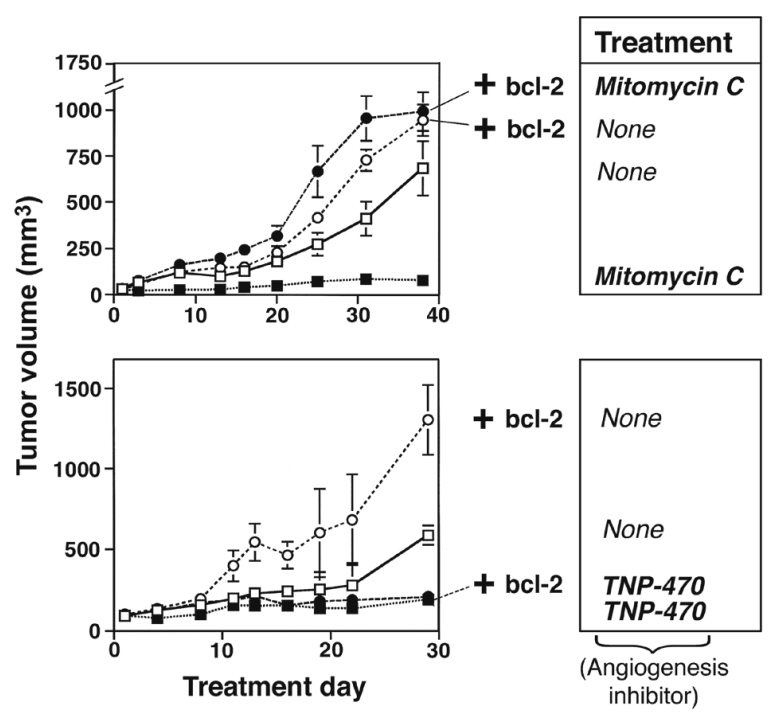

Figure 4. Treatment of human prostate cancer (PC3) in SCID mice, with the angiogenesis inhibitor TNP-470 or the chemotherapeutic agent mitomycin $C$. Tumors derived from the injection of PC3-neo or PC3-bcl-2 were treated with $(A)$ TNP$470(30 \mathrm{mg} / \mathrm{kg}$ every other day subcutaneously) or $(B)$ mitomycin $\mathrm{C} 1 \mathrm{mg} / \mathrm{kg}$ once a week by intraperitoneal injection. ( $\square)$ PC3-neo tumors, untreated; $(\square)$ PC3-neo tumors, treated; $(\bigcirc)$ PC3-bcl-2 tumors, untreated; and (-) PC3-bcl-2 tumors, treated. For each group of tumors, the average volume for five tumors is plotted. Bars, 95\% confidence intervals. (Reprinted, with permission, from Fernandez et al. 2001.)

ation of tumor cells or fibroblasts, and does not inhibit proliferation of the prostate cancer cells. Therefore, oncogene activation (of bcl-2) can be counteracted by blocking angiogenesis, even in a tumor resistant to cytotoxic chemotherapy. In other words, in this experiment, oncogene activation decreased tumor cell apoptosis sufficiently to make the resulting tumor resistant to cytotoxic chemotherapy. In contrast, the increased tumor angiogenesis mediated by oncogene activation could still be completely overridden by nontoxic doses of a broad-spectrum angiogenesis inhibitor.

\section{OVEREXPRESSION OF AN ENDOGENOUS ANGIOGENESIS INHIBITOR CAN COUNTERACT HIGH TUMOR CELL PROLIFERATION AND SUPPRESS TUMOR GROWTH IN VIVO}

There exists additional evidence demonstrating that expansion of tumor mass can vary directly with angiogenesis while tumor cell proliferation remains relatively constant. When the endogenous angiogenesis inhibitors thrombospondin-1 and/or thrombospondin-2 were transfected into a human squamous cell carcinoma (Streit et al. 1999), tumor cells expressed incremental levels of thrombospondin-1 or a combination of thrombospondin-1 and -2 . These cells formed a tumor mass the volume of which varied inversely with thrombospondin-1 expression, and independently of tumor cell proliferation rate (Fig. 5). Increasing thrombospondin-1 expression proportionately decreased tumor angiogenesis and tumor mass, without affecting tumor cell proliferation. 


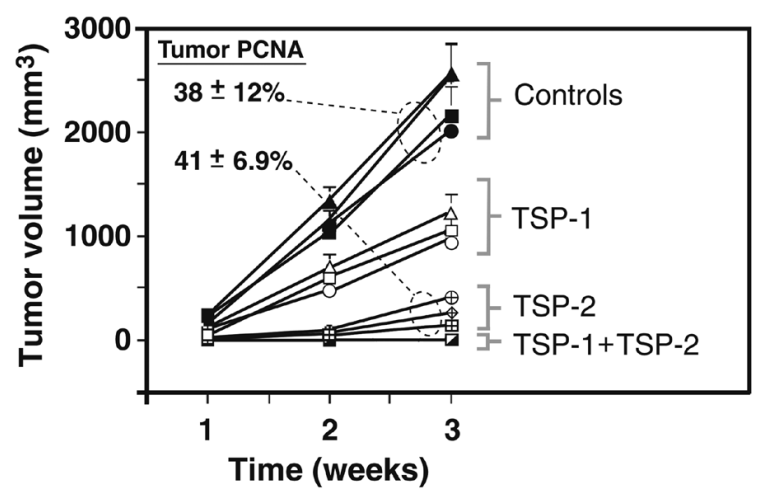

Figure 5. Stable thrombospondin-1 (TSP-1) overexpression in human squamous cell carcinoma cells (A431). Clones expressing increasing levels of TSP-1 significantly inhibited intradermal tumor growth in proportion to TSP-1 expression (i.e., suppressed angiogenesis). Combined overexpression of TSP-1 and TSP-2 completely prevented tumor formation. The largest tumors (controls) had no TSP-1 expression. (Reprinted, with permission, from Streit et al. 1999.)

\section{ONCOGENE ACTIVATION CAN DIRECTLY INCREASE ANGIOGENESIS ACTIVITY OF A TUMOR}

Whereas oncogenes were initially discovered by their ability to increase cell proliferation in vitro, it has only recently been recognized that a major function of oncogenes is to increase tumor angiogenesis. Activated oncogenes can increase angiogenesis either by increasing tumor cell expression of a positive regulator of angiogenesis such as VEGF, or bFGF, or by suppressing expression of an endogenous angiogenesis inhibitor such as thrombospondin-1, or both. Rak et al. (2000) published a series of 15 different oncogenes (Table 1). The list of oncogenes in Table 1 illustrates the extreme heterogeneity of tissues and receptors that are regulated by a relatively small set of pro-angiogenic and anti-angiogenic molecules. The list of oncogenes that regulate angiogenesis continues to grow. Angiogenesis was reported to be an early event in the generation of myc-induced lymphomas (Brandvold et al. 2000). In developing embryos, c-myc is partially responsible for expression of VEGF during vasculogenesis and is also required for the proper expression of angiopoietin-1 and angiopoietin-2 (Baudino et al. 2002). Furthermore, cmyc null embryonic stem cells are dramatically impaired in their ability to form tumors in immune-compromised mice, and the small tumors that sometimes develop are poorly vascularized. Watnick et al. (2003) have demonstrated that in transformed human epithelial cells, the switch to the angiogenic phenotype is mediated by oncogenic Ras suppression of thrombospondin-1. They further show that Ras-dependent repression of thrombospondin-1 is itself dependent on c-myc function, and that this myc function is dependent on phosphorylation of the Myc protein rather than the absolute expression level of myc. Myc phosphorylation is shown to be mediated through ras signaling via phosphatidyl inositol-3 kinase. Therefore, two oncogenes cooperate to turn on angiogenesis in a tumor cell by suppressing an endogenous angiogenesis inhibitor.
Table 1. Impact of Oncogenes or Potential Oncogenes on Angiogenesis Regulatory Proteins

\begin{tabular}{ll}
\hline Oncogene & Implicated pro-angiogenic activity \\
\hline$K$-ras, H-ras & VEGV up-regulation, TSP-1 down-regulation \\
V-src & VEGV up-regulation, TSP-1 down-regulation \\
c-myb & TSP-2 down-regulation \\
N-myc & angiogenic properties in neuroblastoma \\
c-myc & angiogenic properties in epidermis \\
$H E R-2$ & VEGF up-regulation \\
$E G F R$ & VEGF, bFGF, IL-8 up-regulation \\
$P y M T$ & TSP-1 down-regulation \\
c-fos & VEGF expression \\
trkB & VEGF down-regulation \\
$H P V-16$ & secretion of VEGF and IFN- $\alpha$ \\
v- $p 3 k$ & VEGF production and angiogenesis \\
$O D C$ & novel angiogenic factor \\
$P T T G 1$ & VEGF and bFGF up-regulation \\
$E 2 a-P b x 1$ & induction of mouse angiogenin-3 \\
$b c l-2$ & VEGF up-regulation*
\end{tabular}

Data from Rak et al. (2000) and *Fernandez et al. (2001).

Thrombospondin- 1 is only one of at least 28 known endogenous angiogenesis inhibitors that have been discovered in the circulation or in extracellular matrix during the past 20 years (Folkman 2004; Nyberg et al. 2005). It is not clear which of these are possibly downregulated by oncogenes during the switch to the angiogenic phenotype in tumors. However, when endostatin or tumstatin is knocked out, tumor angiogenesis is significantly increased and tumors grow $200-300 \%$ faster. The rate of tumor growth is significantly increased in mice lacking both angiogenesis inhibitors endostatin and tumstatin (Sudhakar et al. 2003).

\section{INACTIVATION OF THE c-myc ONCOGENE INCREASES THROMBOSPONDIN-1 EXPRESSION}

Another finding by Watnick et al. (2003) addresses the question of the mechanism of the rapid regression of a tumor when an oncogene such as Myc is inactivated. In human breast cancer cells, Myc was regulated by the tamoxifen promoter. When the tamoxifen promoter was turned on by estrogen, Myc was inactivated and thrombospondin-1 expression was increased (Fig. 6).

In a collaboration with Dean Felsher's lab, Ryeom and Folkman showed that in Myc-induced lymphoma from the Felsher lab (Felsher and Bishop 1999), thrombospondin-1 was suppressed throughout the growing tumor and its stroma. However, thrombospondin-1 was

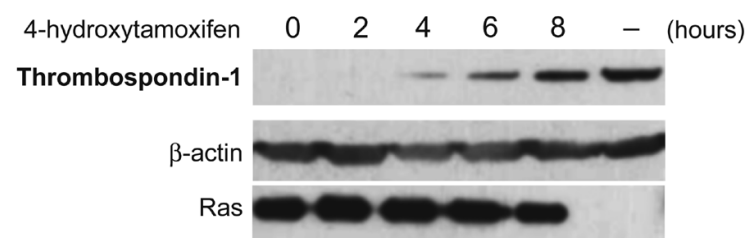

Figure 6. Myc regulation of thrombospondin-1 in human breast cancer cells. Myc is regulated by the tamoxifen promoter in human breast cancer cells. When the tamoxifen promoter is turned on by estrogen, Myc is inactivated and thrombospondin-1 expression is increased. However, upstream molecules are less affected, such as Ras. The loading control is $\beta$-actin. 


\section{MYC-activation induces a growing lymphoma.}

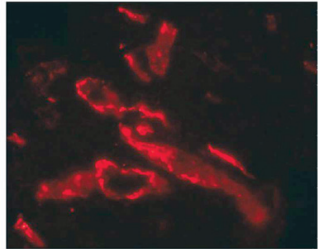

Anti-CD31

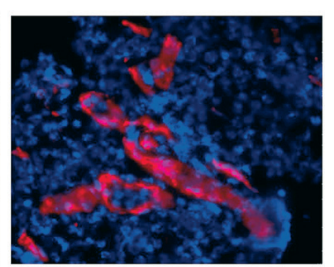

+ Hoechst

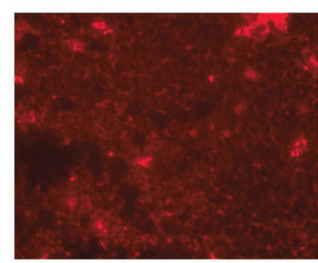

Anti-TSP1

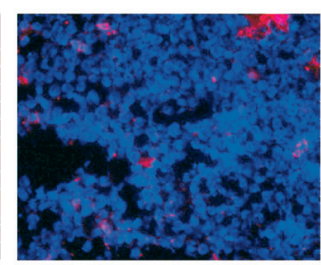

+ Hoechst

\section{MYC-inactivation induces regression of lymphoma.}

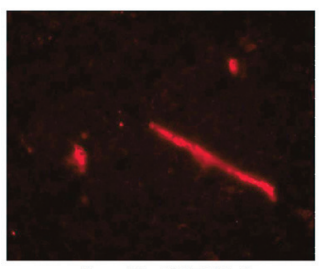

Anti-CD31

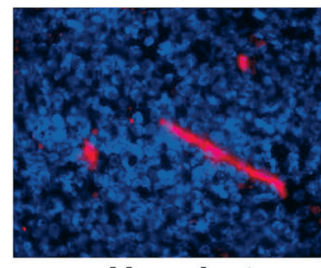

+ Hoechst

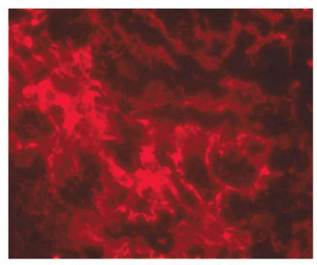

Anti-TSP1

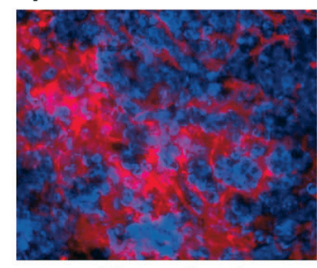

+ Hoechst

Figure 7. Immunohistochemical microsections of a c-myc-activated lymphoma in a mouse. (Upper left panels) Tumor blood vessels stained with anti-CD31 antibody. Intense neovascularization in the tumor bed. Lumens are open. (Upper right panels) Antibody to thrombospondin-1 shows only sparse thrombospondin-1 in the tumor bed. There is residual thrombospondin-1 in the blood vessels. (Lower left panels) Regresson of microvessels in the tumor bed after myc inactivation. Vessels are narrow and closed. (Lower right panels) Abundantly increased thrombospondin-1 has appeared in the tumor bed (S. Giuriato et al., unpubl.).

present in the blood and therefore was visualized in the microvasculature lumen (S. Giuriato et al., unpubl.). Inactivation of c-myc led to tumor regression, which was associated with a dramatic increase in thrombospondin-1 expression throughout the tumor and its stroma (Fig. 7).

\section{TUMOR REGRESSION FROM INACTIVATION OF AN ONCOGENE IS PRECEDED BY IMPAIRMENT OF VASCULAR FUNCTION}

In the tumor model developed by Chin et al. (1999) in which melanoma develops as a result of activation of $\mathrm{H}-$ ras, neovascularization was assessed by in vivo real-time magnetic resonance imaging (Tang et al. 2005). When ras was inactivated, the resulting tumor regression was preceded by impairment of vascular function. This correlated with activation of apoptosis in host-derived endothelial cells as well as in tumor cells. The real-time in vivo imaging provided evidence that loss of vascular integrity upon inactivation of Ras is an active process rather than a consequence of loss of tumor cell viability.

\section{CONCLUSIONS}

Taken together, the evidence assembled here indicates that continuous oncogene activation is necessary to maintain tumor angiogenesis. The angiogenic inducing function of certain oncogenes may operate independently of an oncogene's pro-proliferative effect or antiapoptotic effect on tumor cells.

Activated oncogenes may mediate angiogenesis by upregulating angiogenic proteins (i.e., VEGF) and by downregulating endogenous angiogenesis inhibitors (i.e., thrombospondin-1). A tumor producing high levels of VEGF may not be able to switch to the angiogenic phenotype un- til there has occurred a significant decreased expression of one or more endogenous angiogenesis inhibitors. Tumor cells driven by activated oncogenes may also regulate proangiogenic or antiangiogenic proteins expressed by stromal fibroblasts and/or by vascular endothelium.

Inactivation of an oncogene in a tumor leads to downregulation of pro-angiogenic proteins (i.e., VEGF), upregulation of antiangiogenic proteins (i.e., thrombospondin-1), and rapid vascular regression followed by tumor regression. Oncogene activation can be countered by therapeutic blockade of angiogenesis, even in a tumor that has become resistant to chemotherapy from overexpression of an oncogene such as $b c l-2$. This finding may be important in determining a mechanism for how regressed tumors can escape addiction to a specific oncogene and resume growth (Giuriato and Felsher 2003). Giuriato and Felsher suggest that loss of p53 function could be one explanation. In fact, p53 suppresses angiogenesis by at least four mechanisms: up-regulation of thrombospondin-1 expression (Dameron et al. 1994); degradation of hypoxia inducible factor-1 (Ravi et al. 2000); suppression of VEGF expression (Zhang et al. 2000); and suppression of bFGF-binding protein expression (Sherif et al. 2001). Therefore, as Giuriato and Felsher (2003) emphasize, the loss of p53 function could impede the shutdown of angiogenesis and permit escape of tumor from addiction to a specific oncogene. The list of different angiogenic regulatory molecules that oncogenes encode (Table 1) suggests that tumor escape may not require new mutations, but could simply result from the expansion of a subpopulation of tumor cells that express a different angiogenic protein, bFGF instead of VEGF, for example.

If this phenomenon can be demonstrated in mouse 
Figure 8. $(A)$ Diagram of four classic steps in tumorigenesis relative to the timing of the switch to the angiogenic phenotype during "progression." The switch to the angiogenic phenotype occurs at the stage of microscopic in situ cancer, but is a relatively rare event. By comparing the incidence of in situ cancers found in different organs during autopsies of individuals who died of trauma, but who had no cancer during their lifetime, to the incidence of diagnosed cancer, we estimate that only 1 out of $\sim 600-700$ in situ cancers undergo the angiogenic switch. $(B)$ Diagram to illustrate that by the time a tumor is first diagnosed, it has been growing for several years undetected. The switch to the angiogenic phenotype when a tumor is at $\sim 1 \mathrm{~mm}^{3}$ or less may be among the last barriers that a tumor must overcome before it is potentially lethal. The majority of microscopic sized, in situ cancers don't succeed in becoming angiogenic and remain harmless to the host. If anti-oncogene therapy and/or antiangiogenic therapy could be administered before or soon after the angiogenic switch (e.g., guided by accurate biomarkers), human cancer could possibly be treated before symptoms, or before detection by conventional imaging methods. (A, Modified from Folkman et al. 2006.)

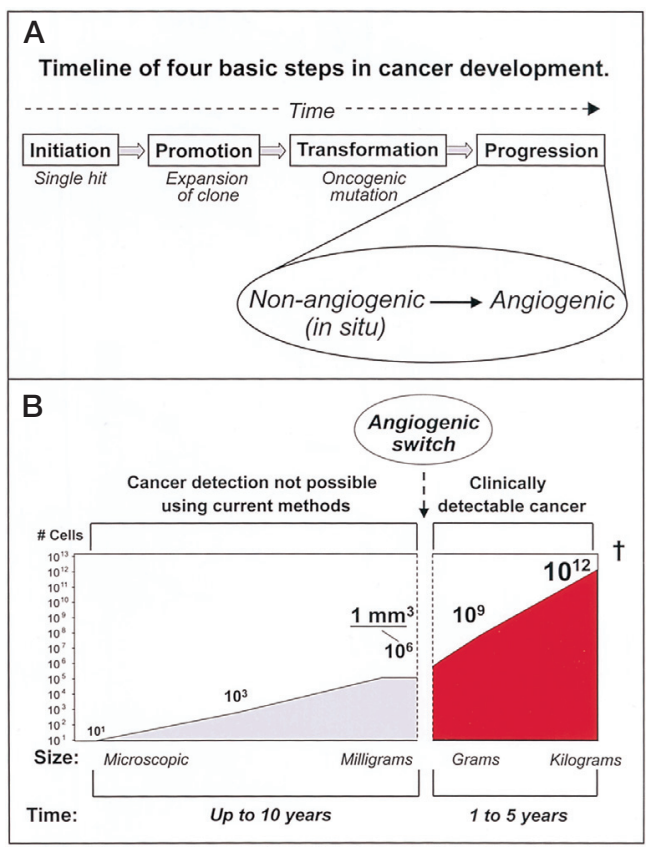

models and if it is found to operate in human tumors in patients, it could explain the occasional escape of tumors from responding to currently available angiogenesis inhibitors after prolonged use (months to more than a year). Most of the angiogenesis inhibitors approved by the FDA to date, and most of those in clinical trials, target only VEGF or its receptors. However, human prostate cancer can express at least four angiogenic proteins, including VEGF, bFGF, interleukin-8, and platelet-derived growth factor (PDGF) (Uehara 2003). Advanced stages of breast cancer can express up to six pro-angiogenic proteins (Relf et al. 1997). In advanced stages of neuroblastoma, up to seven angiogenic proteins are highly expressed (Eggert et al. 2000). If these findings extend to other human cancers, as survival increases, it is likely that residual tumors in a given patient could express redundant angiogenic factors (Folkman 2006). Such tumors could become refractory to an angiogenesis inhibitor that blocks a single angiogenic factor. This potential problem could be circumvented by (1) employing combinations of angiogenesis inhibitors or by (2) combining angiogenesis inhibitors with antiangiogenic chemotherapy (metronomic chemotherapy) (Browder et al. 2000; Klement et al. 2000; Kerbel and Kamen 2004) or by (3) employing broad-spectrum angiogenesis inhibitors that have little or no toxicity, such as endostatin (Folkman 2006) or Caplostatin (Satchi-Fainaro et al. 2005). It is likely that long-term, broad-spectrum antiangiogenic therapy may be desirable to facilitate a goal of "converting cancer to a chronic manageable disease" (Ezzell 1998).

The studies reported here also suggest that antiangiogenic therapy could possibly bypass the problem of a tumor's escape from oncogene addiction, because blockade of angiogenesis blocks tumor growth downstream of oncogene activation (Fig. 8).

\section{HOW COULD THE ANGIOGENESIS DEPENDENCE OF ONCOGENE ADDICTION BE FORMALLY PROVED IN THE FUTURE?}

We have provided indirect evidence that angiogenesis is a requirement for an activated oncogene to induce an expanding tumor mass (including metastasis) (Fig. 9). To provide formal genetic proof of this concept, an oncogene-activated tumor could have the expression of an endogenous angiogenesis inhibitor knocked out, such as thrombospondin-1. These tumors could then be grown in a host null for thrombospondin-1 expression. This model would test whether tumor regression requires either tumor cell production, or host production of angiogenesis inhibitors. The expected outcome could be that oncogene activation would increase tumor growth in the thrombospondin-1 null host as compared to a wild-type tumor in a wild-type host. Therefore, oncogene inactivation could result in a tumor that would

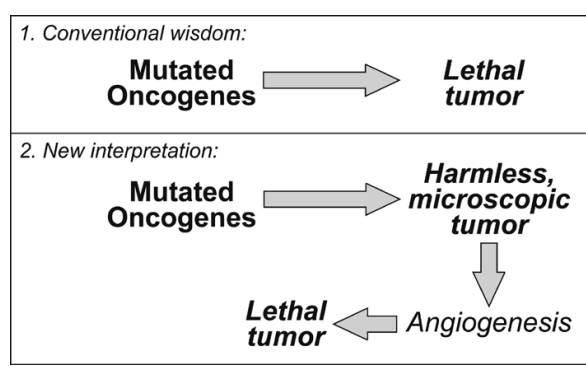

Figure 9. Illustration of the "endothelial centric" view of oncogene addiction. Mutated (activated) oncogenes can transform normal cells to cancer cells, which can grow to a limited microscopic size, i.e., in situ carcinoma. Such a microscopic tumor may remain non-angiogenic, dormant, and harmless, until it can switch to the angiogenic phenotype, after which tumor mass can expand. 
not regress or would regress very slowly. To determine whether this prediction holds up, these experiments are in progress in a collaborative effort between the Felsher and Folkman labs.

\section{ACKNOWLEDGMENTS}

We thank Randolph Watnick for insightful discussions. This work is supported by The Breast Cancer Research Foundation, National Institutes of Health grants RO1 CA064481 and PO1 CA45548 (J.F.), and an Innovator Award from the Department of Defense W81XWH-04-1-0316.

\section{REFERENCES}

Achilles E.G., Fernandez A., Allred E.N., Kisker O., Udagawa T., Beecken W.D., Flynn E., and Folkman J. 2001. Heterogeneity of angiogenic activity in a human liposarcoma: A proposed mechanism for "no take" of human tumors in mice. $J$. Natl. Cancer Inst. 93: 1075.

Almog N., Henke V., Flores L., Hlatky L., Kung A., Wright R.D., Berger R., Hutchinson L., Naumov G., Bender E., Akslen L., Achilles E.G., and Folkman J. 2006. Prolonged dormancy of human liposarcoma is associated with impaired tumor angiogenesis. FASEB J. (in press).

Arbiser J.L., Moses M.A., Fernandez C.A., Ghiso N., Cao Y., Klauber N., Frank D., Brownlee M., Flynn E., Parangi S., Byers H.R., and Folkman J. 1997. Oncogenic H-ras stimulates tumor angiogenesis by two distinct pathways. Proc. Natl. Acad. Sci. 94: 861 .

Auerbach R., Arensman R., Kubai L., and Folkman J. 1975. Tumor-induced angiogenesis: Lack of inhibition by irradiation. Int. J. Cancer 15: 241.

Baudino T.A., McKay C., Pendeville-Samain H., Nilsson J.A., Maclean K.H., White E.L., Davis A.C., Ihle J.N., and Cleveland J.L. 2002. c-Myc is essential for vasculogenesis and angiogenesis during development and tumor progression. Genes Dev. 16: 2530.

Brandvold K.A., Neiman P., and Ruddell A. 2000. Angiogenesis is an early event in the generation of myc-induced lymphomas. Oncogene 19: 2780.

Browder T., Butterfield C.E., Kraling B.M., Shi B., Marshall B., O'Reilly M.S., and Folkman J. 2000. Antiangiogenic scheduling of chemotherapy improves efficacy against experimental drug-resistant cancer. Cancer Res. 60: 1878.

Chin L., Tam A., Pomerantz J., Wong M., Holash J., Bardeesy N., Shen Q., O’Hagan R., Pantginis J., Zhou H., Horner J.W., Cordon-Cardo C., Yancopoulos G.D., and DePinho R.A. 1999. Essential role for oncogenic Ras in tumour maintenance. Nature 400: 468.

Dameron K.M., Volpert O.V., Tainsky M.A., and Bouck N. 1994. Control of angiogenesis in fibroblasts by $\mathrm{p} 53$ regulation of thrombospondin-1. Science 265: 1582.

Eggert A., Ikegaki N., Kwiatkowski J., Zhao H., Brodeur G.M., and Himelstein B.P. 2000. High-level expression of angiogenic factors is associated with advanced tumor stage in human neuroblastomas. Clin. Cancer Res. 6: 1900.

Ezzell C. 1998. Starving tumors of their lifeblood. Sci. Am. 279: 33.

Felsher D.W. and Bishop J.M. 1999. Reversible tumorigenesis by MYC in hematopoietic lineages. Mol. Cell 4: 199.

Fernandez A., Udagawa T., Schwesinger C., Beecken W., Achilles-Gerte E., McDonnell T., and D'Amato R. 2001. Angiogenic potential of prostate carcinoma cells overexpressing Bcl-2. J. Natl. Cancer Inst. 93: 208.

Folkman J. 2000. Tumor angiogenesis. In Cancer medicine, 5th edition (ed. J.F. Holland et al.), p. 132. B.C. Decker, Hamilton, Ontario, Canada.

. 2004. Endogenous angiogenesis inhibitors. Acta Pathol.
Microbiol. Immunol. Scand. 112: 496.

2006. Antiangiogenesis in cancer therapy-endostatin and its mechanisms of action. Exp. Cell Res. 312: 594.

Folkman J. and Kalluri R. 2004. Cancer without disease. Nature 427: 787.

Folkman J., Heymach J., and Kalluri R. 2006. Tumor angiogenesis. In Cancer medicine, 6th edition (ed. D.W. Kufe et al.), p. 157. B.C. Decker, Hamilton, Ontario, Canada.

2004. Cancer without disease. Nature 427: 787.

Giuriato S. and Felsher D.W. 2003. How cancers escape their oncogene habit. Cell Cycle 2: 329.

Hanahan D. and Folkman J. 1996. Patterns and emerging mechanisms of the angiogenic switch during tumorigenesis. Cell 86: 353 .

Holmgren L., O'Reilly M.S., and Folkman J. 1995. Dormancy of micrometastases: Balanced proliferation and apoptosis in the presence of angiogenesis suppression. Nat. Med. 1: 149.

Jain M., Arvanitis C., Chu K., Dewey W., Leonhardt E., Trinh M., Sundberg C.D., Bishop J.M., and Felsher D.W. 2002. Sustained loss of a neoplastic phenotype by brief inactivation of MYC. Science 297: 102.

Karlsson A., Giuriato S., Tang F., Fung-Weier J., Levan G., and Felsher D.W. 2003. Genomically complex lymphomas undergo sustained tumor regression upon MYC inactivation unless they acquire novel chromosomal translocations. Blood 101: 2797.

Kerbel R.S. and Kamen B.A. 2004. The anti-angiogenic basis of metronomic chemotherapy. Nat. Rev. Cancer 4: 423.

Kim K.J., Li B., Winer J., Armanini M., Gillett N., Phillips H.S., and Ferrara N. 1993. Inhibition of vascular endothelial growth factor-induced angiogenesis suppresses tumour growth in vivo. Nature 362: 841.

Klement G., Baruchel S., Rak J., Man S., Clark K., Hicklin D.J., Bohlen P., and Kerbel R. 2000. Continuous low-dose therapy with vinblastine and VEGF receptor-2 antibody induces sustained tumor regression without overt toxicity. J. Clin. Invest. 105: R15.

Naumov G.N., Bender E., Zurakowski D., Kang S.-Y., Sampson D., Flynn E., Watnick R.S., Straume O., Akslen L.A., Folkman J., and Almog N. 2006. A model of human tumor dormancy: An angiogenic switch from the nonangiogenic phenotype. J. Natl. Cancer Inst. 98: 316.

Nyberg P., Xie L., and Kalluri R. 2005. Endogenous inhibitors of angiogenesis. Cancer Res. 65: 3967.

O’Reilly M.S., Boehm T., Shing Y., Fukai N., Vasios G., Lane W.S., Flynn E., Birkhead J.R., Olsen B.R., and Folkman J. 1997. Endostatin: An endogenous inhibitor of angiogenesis and tumor growth. Cell 88: 277 .

O’Reilly M.S., Holmgren L., Shing Y., Chen C., Rosenthal R.A., Moses M., Lane W.S., Cao Y., Sage E.H., and Folkman J. 1994. Angiostatin: A novel angiogenesis inhibitor that mediates the suppression of metastases by a Lewis lung carcinoma. Cell 79: 315.

Rak J., Yu J. L., Klement G., and Kerbel R.S. 2000. Oncogenes and angiogenesis: Signaling three-dimensional tumor growth. J. Investig. Dermatol. Symp. Proc. 5: 24.

Ravi R., Mookerjee B., Bhujwalla Z.M., Sutter C.H., Artemov D., Zeng Q., Dillehay L.E., Madan A., Semenza G.L., and Bedi A. 2000 . Regulation of tumor angiogenesis by p53-induced degradation of hypoxia-inducible factor 1alpha. Genes Dev. 14: 34 .

Relf M., LeJeune S., Scott P.A., Fox S., Smith K., Leek R., Moghaddam A., Whitehouse R., Bicknell R., and Harris A.L. 1997. Expression of the angiogenic factors vascular endothelial cell growth factor, acidic and basic fibroblast growth factor, tumor growth factor beta-1, platelet-derived endothelial cell growth factor, placenta growth factor, and pleiotrophin in human primary breast cancer and its relation to angiogenesis. Cancer Res. 57: 963.

Satchi-Fainaro R., Puder M., Davies J.W., Tran H.T., Sampson D.A., Greene A.K., Corfas G., and Folkman J. 2004. Targeting angiogenesis with a conjugate of HPMA copolymer and TNP-470. Nat. Med. 10: 255.

Satchi-Fainaro R., Mamluk R., Wang L., Short S.M., Nagy J.A., 


\section{ONCOGENE ADDICTION AND ANGIOGENESIS}

Feng D., Dvorak A.M., Dvorak H.F., Puder M., Mukhopadhyay D., and Folkman J. 2005. Inhibition of vessel permeability by TNP-470 and its polymer conjugate, caplostatin. Cancer Cell 7: 251.

Shachaf C.M., Kopelman A.M., Arvanitis C., Karlsson A., Beer S., Mandl S., Bachmann M.H., Borowsky A.D., Ruebner B., Cardiff R.D., Yang Q., Bishop J.M., Contag C.H., and Felsher D.W. 2004. MYC inactivation uncovers pluripotent differentiation and tumour dormancy in hepatocellular cancer. Nature 431: 1112 .

Sherif Z.A., Nakai S., Pirollo K.F., Rait A., and Chang E.H. 2001. Downmodulation of bFGF-binding protein expression following restoration of $\mathrm{p} 53$ function. Cancer Gene Ther. 8: 771.

Streit M., Riccardi L., Velasco P., Brown L.F., Hawighorst T., Bornstein P., and Detmar M. 1999. Thrombospondin-2: A potent endogenous inhibitor of tumor growth and angiogenesis. Proc. Natl. Acad. Sci. 96: 14888.

Sudhakar A., Sugimoto H., Yang C., Lively J., Zeisberg M., and Kalluri R. 2003. Human tumstatin and human endostatin exhibit distinct antiangiogenic activities mediated by alpha $\mathrm{v}$ beta 3 and alpha 5 beta 1 integrins. Proc. Natl. Acad. Sci. 100: 4766 .
Tang Y., Kim M., Carrasco D., Kung A.L., Chin L., and Weissleder R. 2005. In vivo assessment of Ras-dependent maintenance of tumor angiogenesis by real-time magnetic resonance imaging. Cancer Res. 65: 8324.

Udagawa T., Fernandez A., Achilles E.G., Folkman J., and D'Amato R.J. 2002. Persistence of microscopic human cancers in mice: Alterations in the angiogenic balance accompanies loss of tumor dormancy. FASEB J. 16: 1361.

Uehara H. 2003. Angiogenesis of prostate cancer and antiangiogenic therapy. J. Med. Invest. 50: 146.

Volpert O. and Alani R. M. 2003. Wiring the angiogenic switch: Ras, Myc, and Thrombospondin-1. Cancer Cell 3: 199.

Watnick R.S., Cheng Y.-N., Rangarajan A., Ince T.A., and Weinberg R.A. 2003. Ras modulates Myc activity to repress thrombospondin-1 expression and increase tumor angiogenesis. Cancer Cell 3: 219.

Weinstein I.B. 2002. Addiction to oncogenes: The Achilles heal of cancer. Science 297: 63 .

Zhang L., Yu D., Hu M., Xiong S., Lang A., Ellis L.M., and Pollock R.E. 2000. Wild-type p53 suppresses angiogenesis in human leiomyosarcoma and synovial sarcoma by transcriptional suppression of vascular endothelial growth factor expression. Cancer Res. 60: 3655. 


\title{
$\$_{\text {CSH\& }}^{\infty} \mathrm{Cold}$ Spring Harbor Symposia SYMPOSIA on Quantitative Biology
}

\section{Is Oncogene Addiction Angiogenesis-dependent?}

\author{
J. FOLKMAN and S. RYEOM
}

Cold Spring Harb Symp Quant Biol 2005 70: 389-397

Access the most recent version at doi:10.1101/sqb.2005.70.042

$\begin{array}{ll}\text { References } & \begin{array}{l}\text { This article cites } 44 \text { articles, } 15 \text { of which can be accessed free at: } \\ \text { http://symposium.cshlp.org/content/70/389.full.html\#ref-list-1 }\end{array}\end{array}$

\section{License}

Email Alerting Receive free email alerts when new articles cite this article - sign up in Service the box at the top right corner of the article or click here. 